\title{
Absence of EEG gamma coherence in a local activated cortical state: a conserved trait of REM sleep
}

\author{
Matías Cavelli*, Santiago Castro-Zaballa, Alejandra Mondino, Joaquin Gonzalez, Atilio Falconi and Pablo Torterolo \\ Laboratory of Sleep Neurobiology, Department of Physiology, School of Medicine, Universidad de la República, Montevideo, Uruguay
}

\begin{abstract}
During cognitive processes, there are extensive interactions between various regions of the cerebral cortex. Oscillations in the gamma frequency band ( $30-100 \mathrm{~Hz}$ ) of the electroencephalogram are involved in the binding of spatially separated but temporally correlated neural events, which results in a unified perceptual experience.

Like wakefulness, REM sleep is characterized by gamma oscillations in the EEG. Dreams, that are considered a special type of cognitive activity or protoconsciousness, mostly occur during this state.

The power of the gamma band, assessed by the fast Fourier transform, reflects the local degree of synchronization at that frequency. On the other hand, the extent of interactions between different cortical areas at the gamma frequency band can be explored by means of a mathematical function called 'coherence', which reflects the 'strength' of functional interactions between cortical areas.

The objective of the present report was to study in the rat the dynamic relationship between gamma power and coherence in the low $(30-48 \mathrm{~Hz})$ and high $(52-98$ $\mathrm{Hz}$ ) gamma bands during waking and sleep, in occipital, parietal, and frontal neocortical areas, as well as in the olfactory bulb, that is a critical site of gamma rhythmgenesis. In addition, we re-analyzed previous recordings in cats, in order to evaluate the same dynamic relationship as in rats. In both species, the main result was that during REM sleep, gamma power increased, while gamma coherence between distant neocortical areas decreased. The fact that this profile is present in rodenthia as well as in carnivora suggests that this is a trait that characterize REM sleep in mammals.
\end{abstract}

\section{Introduction}

The brain is a complex, self-organized system with non-linear dynamics, in which distributed and parallel processing coexist with serial operations within highly interconnected networks, but without a single coordinating center $[1,2]$. This organ integrates fragmentary neural events that occur at different times and locations into a unified perceptual experience. Understanding the mechanisms that are responsible for this integration, "the binding problem", is one of the most important challenges that cognitive neuroscience has to solve $[3,4]$.

One of the binding mechanisms appears to be the synchronization of neuronal activity by phase-locking of self-generated network oscillations [5,6]. The first experimental evidence supporting the potential role of synchrony as a relational code, was described in simultaneously recorded but spatially segregated neurons that engaged in synchronous oscillation when activated by visual stimuli. The frequency of these synchronized oscillations was in the range of $40 \mathrm{~Hz}[7,8]$. This coordinating mechanisms was named "binding-bysynchrony" [2,4]; and this theory assumes that coherence in neuronal activity is critical for information processing [9].

Jasper and Andrews first used the term gamma waves to designate low-amplitude waves at $35-45 \mathrm{~Hz}$ in the electroencephalogram (EEG) [10]. These oscillations were later described in the olfactory bulb (OB) of hedgehogs by Adrian [11]. An increase in gamma activity typically appears during states/behaviors that are characterized by the active cognitive processing of external percepts or internally generated thoughts and images [12-16].

Cognitive activities not only occur during wakefulness (W). Dreams, which occur mostly during REM sleep, are considered a special kind of cognitive activity or proto-consciousness [17]. In contrast, during deep non-REM (NREM) sleep, there is an absence, or at least a strong reduction, in oneiric activity [17]. REM sleep dreams are characterized by their vividness, single-mindedness, bizarreness and loss of voluntary control over the plot. Attention is unstable and rigidly focused, facts and reality are not checked, violation of physical laws and bizarreness are passively accepted, contextual congruence is distorted, time is altered and memories become labile $[17,18]$. Interestingly, some authors have suggested that cognition during REM sleep resembles psychosis [19]. High gamma activity is also present during REM sleep, both in humans and animals [20-23].

Local cortical oscillations in the gamma band can be examined by means of the fast Fourier transform (FFT). The results are expressed in gamma power that reflects the local degree of synchronization of the extracellular potential at that frequency [24]. Local gamma oscillations are higher during REM sleep compared to NREM sleep [21-23,25]. On the other hand, the extent of interactions between different cortices at the gamma frequency band can be explored through a mathematical function called 'coherence', which reflects the 'strength' of functional interactions between cortical areas [26-28]. Gamma coherence between distant areas has been proposed as a neural correlate of

Correspondence to: Dr. Pablo Torterolo, Department of Physiology, Faculty of Medicine, University of the Republic, General Flores 2125, 11800 MontevideoUruguay, Tel: (598) 29243414 ext. 3234; E-mail: ptortero@fmed.edu.uy

Key words: wakefulness, paradoxical sleep, cortex, dreams, synchronization, NREM sleep

Received: June 29, 2017; Accepted: July 28, 2017; Published: July 31, 2017 
conscious perception and self-awareness [29-33]. In this regard, coherence in the gamma frequency band is lost during anesthesiainduced unconsciousness [34-36], and is severely altered in psychiatric disorders $[37,38]$.

In previous studies, we showed in cats and rats, that gamma coherence is high during $\mathrm{W}$, decreases during NREM sleep and is almost absent during REM sleep [23,39-41]. However, these studies were limited in the extent of the cortical loci studied, as well as in the examination of the dynamic relationship between local and long-range gamma synchronization. Therefore, the aim of the present report was the following: 1 . To study in the rat low $(30-48 \mathrm{~Hz})$ and high $(52-98$ $\mathrm{Hz}$ ) gamma power and coherence during $\mathrm{W}$ and sleep in neocortical areas (such as frontal cortices) that were not yet studied. We also aimed to analyze the $\mathrm{OB}$, that is a critical site of gamma rhythm-genesis $[11,14,15]$. In addition, we evaluated in detail the dynamic relationship between local or short-range synchronization (power) and long-range synchronization (coherence) during $\mathrm{W}$, sleep and transitions into and out REM sleep. 2. To re-analyze previous recordings in cats, in order to evaluate the same dynamic relationships proposed for rats.

\section{Material and methods}

\section{Experimental animals}

We analyzed data obtained from nine adult Wistar rats and six adult cats. Data from four of these cats were utilized in previous studies $[39,41]$. The animals were determined to be in good health by veterinarians of the Department of Laboratory Medicine of the School of Medicine, Universidad de la República, Uruguay. All experimental procedures were conducted in accordance with the National Animal Care Law (\#18611) and with the "Guide to the care and use of laboratory animals" (8th edition, National Academy Press, Washington D. C., 2010). Furthermore, the Institutional Animal Care Committee approved the experimental procedures. Adequate measures were taken to minimize pain, discomfort or stress of the animals, and efforts were made to use the minimum number of animals necessary to produce reliable data.

Surgical procedures and experimental sessions are summarized below; for details $[23,39]$.

\section{Surgical procedures}

Animals were chronically implanted with electrodes to monitor the states of sleep and W. In rats, anesthesia was induced with a mixture of ketamine-xylazine. Cats were pre-medicated with xylazine, atropine and antibiotics, and anesthesia was induced with ketamine and maintained with a gas mixture of isoflourane in oxygen.

The animal's head was positioned in a stereotaxic frame and the skull was exposed. In order to record the EEG, stainless steel screw electrodes (diameter: $1.4 \mathrm{~mm}$ cats and $1.0 \mathrm{~mm}$ for rats) were placed on the surface (above the dura mater) in different cortices. Figure 1 presents a summary of the positions of the recording electrodes on the surface of the cortex for rats and cats. In the rats, six electrodes were located on the neocortex forming two anterior-posterior consecutive squares centered with respect to the midline and the frontal square centered with respect to Bregma (Figure 1). Each side of the squares has a length of $5 \mathrm{~mm}$. The electrodes were located in primary motor cortex (M1, L: $\pm 2.5 \mathrm{~mm}$, AP: $+2.5 \mathrm{~mm}$ ), primary somato-sensory cortex (S1, L: $\pm 2.5 \mathrm{~mm}$, AP: $-2.5 \mathrm{~mm}$ ), and secondary visual cortex (V2, L: $\pm 2.5 \mathrm{~mm}$, AP: $-7.5 \mathrm{~mm}$ ). The other electrode was located over the right $\mathrm{OB}(\mathrm{L}:+1.25 \mathrm{~mm}$, AP: $+7.5 \mathrm{~mm})$. Each cat was implanted with several electrodes throughout the neocortex. However, in this


CAT

Figure 1. Position of recording electrodes. The figure presents a summary of the positions of the recording sites on the surface of the cerebral cortex of a group of 9 rats sharing the same location of electrodes, and 6 cats with similar but not the same electrode locations as in rats. The electrodes were referred to a common electrode that was located over the cerebellum (rats) or left frontal sinus (cats). OB, olfactory bulb; M1, primary motor cortex; $\mathrm{S} 1$, primary somato-sensory cortex; V1, primary visual cortex; V2, secondary visual cortex; Pf, pre-frontal cortex; Pp, posterior parietal cortex.

report, we analyzed the signals from the electrodes positioned in the anterior (frontal) and posterior (occipital or parietal) cortices of the same hemisphere.

The electrodes were soldered to a plug and bonded to the skull with acrylic cement. In order to record the electromyogram (EMG), two electrodes were inserted into the neck muscle chronically in the rat, and contact electrodes (with EEG-paste interphase) were acutely positioned on the neck skin of the cat. In the cat, two plastic tubes (which were used to maintain the animal's head fixed without pain or pressure), were also bonded to the skull with acrylic cement.

At the end of the surgical procedures, an analgesic was administered. Incision margins were kept clean and a topical antibiotic was administered on a daily basis. After the animals had recovered from the preceding surgical procedures, they were adapted to the recording environment for a period of at least one week (rats) and two months (cats).

\section{Experimental sessions}

Experimental sessions of 4-6 h in duration were conducted during the light period, between $12 \mathrm{AM}$ and $6 \mathrm{PM}$ (rats) and $11 \mathrm{AM}$ to $3 \mathrm{PM}$ (cats) in a temperature-controlled environment $\left(21-24{ }^{\circ} \mathrm{C}\right)$. In rats, the recordings were obtained via a rotating connector in a soundattenuated chamber which is also a Faraday box; during these sessions (as well as during adaptation sessions), the animals were able to move freely within the confines of the recording chamber (transparent cages $(40 \times 30 \times 20 \mathrm{~cm})$ containing wood shavings) and had free access to water and food. Cats were recorded in a Faraday box in semi-restricted conditions; during experimental sessions' the head of the cat was held in a stereotaxic frame by a head-restraining device, while the body rested in a sleeping bag.

The simultaneous activity of different cortical areas was recorded with monopolar arrangement of electrodes [42]. A common electrode reference montage was employed; it was located in the left frontal sinus of the cats [39], and in the cerebellum of the rats [23]. The EMG was also monitored. Each cat and rat was recorded daily for a period of approximately 30 days and 2 weeks, respectively, in order to obtain a complete data set.

Bioelectric analog signals were amplified with differential AC amplifiers (AM-systems model 1700; $1000 \mathrm{x}$ ), filtered $(0.1-500 \mathrm{~Hz}$ ), digitized $\left(1024 \mathrm{~Hz}, 2^{16}\right.$ bits) and stored on a PC using the Spike2 software (Cambridge Electronic Design). Data were obtained during W, REM sleep, and NREM sleep. 


\section{Data analysis}

Sleep and waking states were determined for every 10-seconds epoch for rats and cats. W, light sleep (SL1), deep or slow wave sleep (SL2), NREM sleep (SL1 + SL2) and REM sleep were identified in rats $[23,43]$. Alert W (AW), quiet W $(\mathrm{QW}), \mathrm{W}(\mathrm{AW}+\mathrm{QW})$, NREM sleep and REM sleep were identified in cats [39]. AW was induced for a period of $300 \mathrm{sec}$ by a sound stimulus, which was introduced approximately 30 minutes after the beginning of the recording. The sound stimulus consisted of clicks ( $0.1 \mathrm{~ms}$ in duration) of 60 to $100 \mathrm{~dB}$ SPL in intensity with a variable frequency of presentation $(1$ to $500 \mathrm{~Hz}$, modified at random by the operator) in order to avoid habituation [39].

We focused on the analysis of low (LG: $30-48 \mathrm{~Hz}$ ) and high (HG: $52-98 \mathrm{~Hz}$ ) gamma frequency bands of the EEG; $50 \mathrm{~Hz}$ electrical noise was avoided with this partitioning. In rats, we analyzed gamma power in the maximum number of channels and coherence in all the possible cortical combinations. In cats, we focused on a pair of electrodes with similar location in all animals, one in a frontal position and one in a posterior position of the brain. In order to analyze gamma power and gamma coherence between these EEG channels, we used similar procedures as in our previous studies $[23,39,41]$; however, the analysis in cats and rats were not exactly the same (see below).

\section{CAT}

We employed the same methodology that we described in our previous studies [39-41]. Twelve artifact-free periods of 100 seconds were examined during each behavioral state (1200 seconds for each behavioral state, per animal; data were selected from three different recordings).

\section{RAT}

The maximum number of non-transitional and artifact-free periods of 30 seconds was selected during each behavioral state to determine the mean power and coherence for each rat. For each animal, we analyzed two complete $(6 \mathrm{~h})$ recordings (recordings with the minimal amount of artifacts were selected).

The coherence between two EEG channels that were recorded simultaneously was analyzed in windows of 30 second (rat) or 100 second (cat) windows. For each period, the Magnitude Squared Coherence as well as the power spectrum for each channel, were calculated by means of Spike2 script COHER-HOL 1S (for details about coherence definition see [26,39]). For the coherence analysis, each period was divided into 30 (rat) and 100 (cat) time-blocks with a bin size of 2048 samples, and a resolution of $0.5 \mathrm{~Hz}$. We applied the Fisher z' transform to the gamma coherence values in order to normalize and evaluate them by means of parametric statistical tests. The data was then analyzed through custom-built Python routines.

Analyses of 10-second epochs were also used to determine the temporal dynamic of power and coherence. Hence, low and high gamma power and coherence were normalized, and referred to as normalized power (NP) and normalized z'-coherence (NC). For dynamic analysis (see Results), normalization was undertaken by dividing each value by the maximum value (of either power or coherence) recorded in each analyzed segment. For statistical analysis, the normalization was performed for each animal by dividing each mean value by the maximum-recorded value.

We also analyzed the LG and HG mean global power (MGP) and mean global coherence (MGC) in each rat, by averaging the power measured in all the channels, and the coherence for all combinations of electrodes [36].
In order to determine the relationship between local or short-range synchronization (power) and long-range synchronization (coherence) we applied the following function: ((NP of Ch1 + NP Ch2) / 2)) (NC between Ch1-Ch2). We named this function Power-Coherence difference (NP-NC). A similar approach was employed for the global power and coherence (MGP - MGC). In previous preliminary analyses, we constructed the function utilizing the ratio between normalized power and coherence with similar results. However, we considered the NP-NC function a more suitable analysis given that the signs of the results differed between states; W (close to zero), NREM sleep (negative values) and REM sleep (positive values).

The data were expressed as mean \pm standard deviation of the NP, $\mathrm{NC}$ and NP-NC. The significance of the differences across behavioral states was evaluated with one-way ANOVA and Tamhane post hoc tests (for each animal) and repeated measures ANOVA (for comparison between the means of the whole group of cats or rats), along with Tukey post hoc tests. The criterion used to reject the null hypothesis was $\mathrm{p}<0.05$.

\section{Results}

\section{Gamma activity in rat}

Dynamics of gamma activity in the rat: The gamma dynamic during sleep and $\mathrm{W}$ in a representative rat is shown in Figure 2; the spectrograms and hypnograms of Figure 2A, indicate the different states of $\mathrm{W}$ and sleep. Low and high gamma NP is shown in Figure 2B. For primary somatosensory (S1) and secondary visual (V2) cortices, both low and high gamma NP was higher during $\mathrm{W}$ than during sleep. For the low gamma band in this animal there were not clear changes in the NP between NREM sleep and REM sleep. On the contrary, high gamma NP was minimal in NREM sleep but increased during REM sleep (Figure 2B).

Low and high gamma NC between S1 and V2 were the highest during $\mathrm{W}$ and decreased during NREM sleep (Figure 2C). The largest decrement in low and high gamma NC occurred during REM sleep.

In order to analyze the relationship between gamma power (that reflects local or short-range synchronization) and gamma coherence (that reveals long-range or distant synchronization), the NP-NC function was applied (Figure 2C). During W this relationship was close to zero, had negative values during NREM sleep and reverted to positive values during REM sleep. This dynamic profile was similar for both low and high gamma bands.

Cortical extent of LG and HG power and coherence: To study the cortical extent of power and coherence of gamma bands, we analyzed a group of nine rats that shared the same electrode positions. Statistical analyses of gamma power and coherence are shown in Tables 1,2 and 3; these results are graphically summarized in Figure 3. LG and HG NP showed significant differences across behavioral states in all the cortical regions (Table 1). These results revealed that LG and HG power is high during $\mathrm{W}$ than during sleep, both in neocortex and OB. The lowest values were recorded during NREM sleep, while during REM sleep the HG NP increased to an intermediate level in all the cerebral areas recorded. On the other hand, LG showed similar values to those of $\mathrm{W}$ in the parietal and frontal areas of the neocortex (Table 1).

For LG, NC displayed significant differences across behavioral states from sixteen of twenty-one possible electrode pair combinations (Table 2). Interestingly, seven combinations displayed a greater coherence during NREM sleep than during W. In contrast, only two 


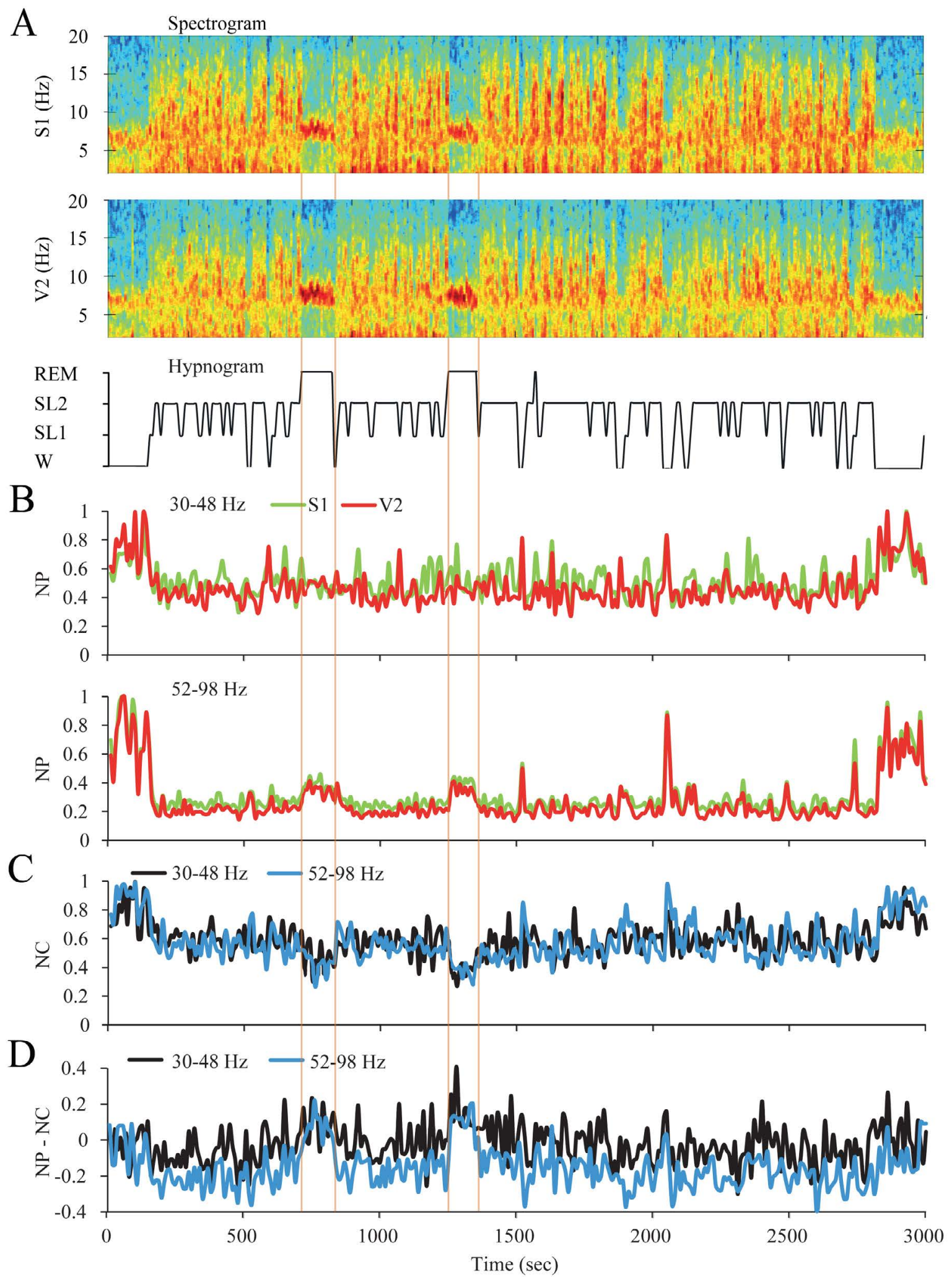

Figure 2. Dynamic of the gamma activity in the rat. A. The spectrograms (1-20 Hz) of secondary visual (V2) and primary somatosensory (S1) cortical recordings and the hypnogram are shown. During W and REM sleep, theta activity (4-9 Hz) in the spectrograms can be readily observed. During NREM sleep, delta activity (0.5-4 Hz) was more prominent and there were intermittent episodes of sigma activity $(9-15 \mathrm{~Hz})$, which correspond to the presence of sleep spindles. Color calibration of the spectrogram is not shown. B. Normalized power $(\mathrm{NP})$ of $\mathrm{S} 1$ (green) and V1 (red) cortices for low (upper traces) and high (lower traces) gamma bands are shown. C. Normalized z'-coherence (NC) for both gamma bands (30-48 and 52-98 Hz). D. Power-coherence difference function (NP-NC) discriminates between REM sleep with positive values and the rest of states with zero or negative values. All the parameters were analyzed in 10 second epochs. W, wakefulness; SL1, light sleep; SL2, slow wave sleep; REM, REM sleep. 
Table 1. Normalized power during sleep and wakefulness in the rat.

\begin{tabular}{|c|c|c|c|c|c|c|c|c|c|}
\hline \multirow[b]{2}{*}{ Band } & \multirow[b]{2}{*}{ Electrode } & \multicolumn{3}{|c|}{ Descriptive Statistics (NP) } & \multicolumn{2}{|c|}{ Repeated measures ANOVA } & \multicolumn{3}{|c|}{ Multiple Comparisons-Tukey } \\
\hline & & $\mathrm{W}($ mean $\pm \mathrm{SD})$ & $\operatorname{NREM}($ mean \pm SD) & $\operatorname{REM}($ mean \pm SD) & $\mathrm{F}$ & Sig. (p) & W vs NREM (p) & W vs REM (p) & REM vs NREM (p) \\
\hline \multirow{7}{*}{ LG } & $\mathrm{OBr}$ & $1.0 \pm 0.0$ & $0.48 \pm 0.05$ & $0.84 \pm 0.10$ & 142.7 & $<0.001$ & $<0.001$ & $<0.001$ & $<0.001$ \\
\hline & M11 & $0.89 \pm 0.13$ & $0.51 \pm 0.11$ & $0.98 \pm 0.03$ & 70.9 & $<0.001$ & $<0.001$ & 0.122 & $<0.001$ \\
\hline & M1r & $0.89 \pm 0.12$ & $0.52 \pm 0.08$ & $0.97 \pm 0.04$ & 71.3 & $<0.001$ & $<0.001$ & 0.130 & $<0.001$ \\
\hline & S11 & $0.86 \pm 0.15$ & $0.64 \pm 0.18$ & $0.95 \pm 0.07$ & 13.9 & $<0.001$ & 0.007 & 0.263 & $<0.001$ \\
\hline & S1r & $0.91 \pm 0.15$ & $0.71 \pm 0.16$ & $0.96 \pm 0.06$ & 12.1 & $<0.000$ & 0.004 & 0.691 & $<0.001$ \\
\hline & V21 & $1.0 \pm 0.0$ & $0.57 \pm 0.18$ & $0.81 \pm 0.13$ & 38.1 & $<0.000$ & $<0.000$ & 0.003 & $<0.000$ \\
\hline & $\mathrm{V} 2 \mathrm{r}$ & $0.98 \pm 0.03$ & $0.57 \pm 0.14$ & $0.78 \pm 0.13$ & 36.3 & $<0.000$ & $<0.000$ & 0.001 & 0.001 \\
\hline \multirow{7}{*}{$\mathrm{HG}$} & OBl & $1.0 \pm 0.0$ & $0.32 \pm 0.10$ & $0.44 \pm 0.12$ & 190.1 & $<0.001$ & $<0.001$ & $<0.001$ & 0.001 \\
\hline & M1r & $0.99 \pm 0.01$ & $0.33 \pm 0.08$ & $0.77 \pm 0.16$ & 69.8 & $<0.001$ & $<0.001$ & 0.003 & $<0.001$ \\
\hline & M11 & $0.99 \pm 0.01$ & $0.36 \pm 0.10$ & $0.78 \pm 0.12$ & 98.6 & $<0.001$ & $<0.001$ & $<0.001$ & $<0.001$ \\
\hline & S1r & $0.99 \pm 0.01$ & $0.49 \pm 0.10$ & $0.87 \pm 0.08$ & 106.3 & $<0.001$ & $<0.001$ & 0.007 & $<0.001$ \\
\hline & S11 & $0.99 \pm 0.00$ & $0.53 \pm 0.09$ & $0.89 \pm 0.06$ & 129.1 & $<0.001$ & $<0.001$ & 0.008 & $<0.001$ \\
\hline & $\mathrm{V} 2 \mathrm{r}$ & $0.99 \pm 0.02$ & $0.45 \pm 0.15$ & $0.74 \pm 0.19$ & 46.8 & $<0.001$ & $<0.001$ & 0.001 & $<0.001$ \\
\hline & V2d & $0.99 \pm 0.01$ & $0.38 \pm 0.17$ & $0.63 \pm 0.27$ & 35.4 & $<0.001$ & $<0.001$ & $<0.001$ & 0.011 \\
\hline
\end{tabular}

The analysis was performed in 9 animals. The degrees of freedom were 2 (between groups) and 16 (within groups). OB, olfactory bulb; M1, primary motor cortex; S1, primary somatosensory cortex; V2, secondary visual cortex r, right; 1, left. W, wakefulness; NREM, non-REM sleep; REM, REM sleep. *, p $<0.05$.

Table 2. LG normalized coherence during sleep and wakefulness in the rat.

\begin{tabular}{|c|c|c|c|c|c|c|c|c|}
\hline \multirow[b]{2}{*}{ Electrode pairs } & \multicolumn{3}{|c|}{ Descriptive Statistics (NC) } & \multicolumn{2}{|c|}{ Repeated measures ANOVA } & \multicolumn{3}{|c|}{ Multiple Comparisons-Tukey } \\
\hline & $\mathrm{W}($ mean $\pm \mathrm{SD})$ & $\operatorname{NREM}($ mean \pm SD $)$ & $\operatorname{REM}($ mean $\pm \mathrm{SD})$ & $\mathrm{F}$ & Sig. (p) & W vs SWS (p) & W vs REM (p) & REM vs NREM (p) \\
\hline OBr-M1r & $0.91 \pm 0.09$ & $0.86 \pm 0.12$ & $0.92 \pm 0.07$ & 0.9 & 0.409 & 0.506 & 0.994 & 0.449 \\
\hline OBr-M11 & $0.93 \pm 0.08$ & $0.86 \pm 0.12$ & $0.93 \pm 0.07$ & 1.6 & 0.225 & 0.285 & 0.999 & 0.289 \\
\hline $\mathrm{OBr}-\mathrm{S} 1 \mathrm{r}$ & $0.93 \pm 0.07$ & $0.79 \pm 0.17$ & $0.82 \pm 0.16$ & 2.2 & 0.135 & 0.138 & 0.281 & 0.898 \\
\hline OBr-S11 & $0.93 \pm 0.07$ & $0.79 \pm 0.15$ & $0.85 \pm 0.14$ & 2.4 & 0.121 & 0.105 & 0.401 & 0.674 \\
\hline $\mathrm{OBr}-\mathrm{V} 2 \mathrm{r}$ & $0.93 \pm 0.09$ & $0.90 \pm 0.07$ & $0.81 \pm 0.16$ & 2.4 & 0.120 & 0.870 & 0.119 & 0.272 \\
\hline OBr-V21 & $0.90 \pm 0.09$ & $0.96 \pm 0.06$ & $0.83 \pm 0.12$ & 4.2 & 0.033 & 0.370 & 0.304 & 0.025 \\
\hline M1r-M11 & $0.98 \pm 0.02$ & $0.95 \pm 0.05$ & $0.81 \pm 0.09$ & 17.4 & $<0.001$ & 0.480 & $<0.001$ & 0.001 \\
\hline M1r-S1r & $0.98 \pm 0.03$ & $0.90 \pm 0.05$ & $0.91 \pm 0.06$ & 4.4 & 0.030 & 0.041 & 0.066 & 0.965 \\
\hline M1r-S11 & $0.97 \pm 0.04$ & $0.90 \pm 0.09$ & $0.80 \pm 0.09$ & 11.8 & $<0.001$ & 0,105 & $<0.001$ & 0.042 \\
\hline M1r-V2r & $0.94 \pm 0.09$ & $0.94 \pm 0.06$ & $0.69 \pm 0.12$ & 17.7 & $<0.001$ & 0,991 & $<0.001$ & $<0.001$ \\
\hline M1r-V21 & $0.88 \pm 0.08$ & $0.99 \pm 0.01$ & $0.68 \pm 0.10$ & 31.7 & $<0.001$ & 0.031 & $<0.001$ & $<0.001$ \\
\hline M11-S11 & $0.97 \pm 0.03$ & $0.88 \pm 0.05$ & $0.92 \pm 0.07$ & 4.6 & 0.025 & 0.019 & 0.275 & 0.333 \\
\hline M11-V21 & $0.85 \pm 0.09$ & $0.99 \pm 0.01$ & $0.66 \pm 0.11$ & 35.3 & $<0.001$ & $<0.001$ & $<0.001$ & $<0.001$ \\
\hline S1r-M11 & $0.98 \pm 0.03$ & $0.93 \pm 0.06$ & $0.79 \pm 0.09$ & 20.4 & $<0.001$ & 0.296 & $<0.001$ & $<0.001$ \\
\hline S1r-S11 & $0.94 \pm 0.06$ & $0.97 \pm 0.03$ & $0.79 \pm 0.10$ & 17.7 & $<0.001$ & 0.682 & $<0.001$ & $<0.001$ \\
\hline S1r-V21 & $0.69 \pm 0.13$ & $1.0 \pm 0.0$ & $0.59 \pm 0.12$ & 77.9 & $<0.001$ & $<0.001$ & 0.020 & $<0.001$ \\
\hline V2r-M11 & $0.89 \pm 0.13$ & $0.96 \pm 0.05$ & $0.73 \pm 0.17$ & 8.41 & 0.003 & 0.544 & 0.027 & 0.003 \\
\hline V2r-S11 & $0.77 \pm 0.13$ & $1.0 \pm 0.0$ & $0.63 \pm 0.17$ & 27.1 & $<0.001$ & $<0.001$ & 0.041 & $<0.001$ \\
\hline V2r-V21 & $0.61 \pm 0.09$ & $1.0 \pm 0.0$ & $0.55 \pm 0.12$ & 91.4 & $<0.001$ & $<0.001$ & 0.269 & $<0.001$ \\
\hline S11-V21 & $0.81 \pm 0.11$ & $1.0 \pm 0.0$ & $0.71 \pm 0.06$ & 50.1 & $<0.001$ & $<0.001$ & 0.005 & $<0.001$ \\
\hline S1r-V2r & $0.81 \pm 0.13$ & $0.98 \pm 0.03$ & $0.75 \pm 0.15$ & 11.7 & $<0.001$ & 0.009 & 0.443 & $<0.001$ \\
\hline
\end{tabular}

The analysis was performed in 9 animals. The degrees of freedom were 2 (between groups) and 16 (within groups). OB, olfactory bulb; M1, primary motor cortex; S1, primary somatosensory cortex; V2, secondary visual cortex r, right; 1, left. W, wakefulness; NREM, non-REM sleep; REM, REM sleep. *, p $<0.05$.

electrode combinations showed the opposite pattern. In adition, NC during $\mathrm{W}$ was higher than during REM sleep for eleven combinations while in REM sleep NC was lower than during NREM sleep in fourteen electrode combinations. Interestingly, LG coherence between most of the $\mathrm{OB}$ and neocortical combinations were not modified across behavioral states.

For HG, NC also displayed significant differences across behavioral states for nineteen out of twenty-one combinations (Table 3). Coherence was higher during $W$ than during NREM sleep in eighteen combinations, and higher than during REM sleep in nineteen combinations. REM sleep showed lower NC than during NREM sleep in nine combinations of cortical electrodes.

Mean global gamma activity during sleep and wakefulness in the rat: Figure 4A illustrates the MGP, MGC and their difference in nine rats, during the $\mathrm{W}$ and sleep. Gamma MGP showed significant differences across behavioral states $\left(\mathrm{LG}, \mathrm{F}_{2,16}=58.1, \mathrm{p}<0.001\right.$; HG, $\left.\mathrm{F}_{2,16}=178.1, \mathrm{p}<0.001\right)$. Figure $4 \mathrm{~A}$ reveals that LG MGP did not present significant differences between $\mathrm{W}$ and REM sleep. Both during $\mathrm{W}$ and REM sleep, LG MGP was higher than during NREM sleep. On the other hand, HG MGP was highest during $\mathrm{W}$, had an intermediate value during REM sleep and reached the minimum value during NREM sleep.

There was a significant difference in MGC across behavioral states (LG, $\mathrm{F}_{2,16}=26.6, \mathrm{p}<0.001 ; \mathrm{HG}, \mathrm{F}_{2,16}=86.7, \mathrm{p}<0.001$ ). Figure $4 \mathrm{~B}$ shows that LG MGC did not present significant differences between $\mathrm{W}$ and NREM sleep. The lowest values of low and high gamma coherence were present during REM sleep (Figure 4B).

NP-NC was significantly different for low and high gamma bands (LG, $\mathrm{F}_{2,16}=97.1, \mathrm{p}<0.001 ; \mathrm{HG}, \mathrm{F}_{2,16}=55.3, \mathrm{p}<0.001$ ). During $\mathrm{W}$, the 
HG W - NREM

mean difference

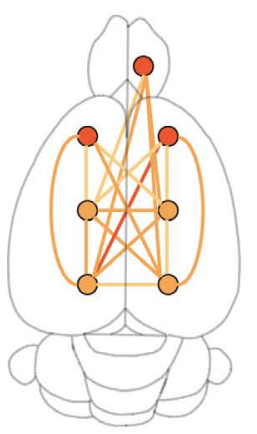

LG W - NREM

mean difference



HG W - REM

mean difference

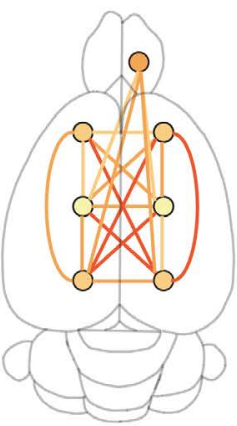

LG W - REM

mean difference

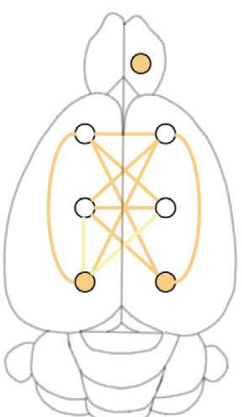

HG REM - NREM

mean difference

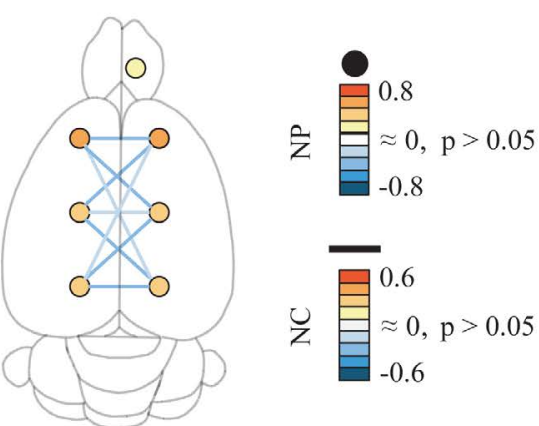

Figure 3. Low (LG) and high gamma (HG) band power and coherence in the rat. Summary of the statistically significant differences between behavioral states of the LG and HG normalized power (NP) and normalized coherence (NC) over the surface of the cerebral cortex. The circles represent the power for the different cortical regions, while the lines represent the coherence for the different combinations of electrodes. The colors represent the mean difference level of NP and NC. Data were obtained from the mean values of all available, nontransitional artifact-free windows per rat for each behavioral state ( 9 rats). Repeated mesures ANOVA and Tukey paired comparisons tests. OB, olfactory bulb; M1, primary motor cortex; S1, primary somato-sensory cortex; secondary V2, visual cortex.

Table 3. HG normalized coherence during sleep and wakefulness in the rat.

\begin{tabular}{|c|c|c|c|c|c|c|c|c|}
\hline \multirow[b]{2}{*}{ Electrode pairs } & \multicolumn{3}{|c|}{ Descriptive Statistics (NC) } & \multicolumn{2}{|c|}{ Repeated measures ANOVA } & \multicolumn{3}{|c|}{ Multiple Comparisons-Tukey } \\
\hline & $\mathrm{W}($ mean $\pm \mathrm{SD})$ & $\operatorname{NREM}($ mean $\pm \mathrm{SD})$ & REM (mean $\pm \mathrm{SD})$ & $\mathrm{F}$ & Sig. (p) & W vs SWS (p) & W vs REM (p) & REM vs NREM (p) \\
\hline OBr-M1r & $0.93 \pm 0.10$ & $0.76 \pm 0.16$ & $0.83 \pm 0.16$ & 2.9 & 0.081 & 0.0686 & 0.363 & 0.580 \\
\hline OBr-M11 & $0.93 \pm 0.09$ & $0.77 \pm 0.12$ & $0.84 \pm 0.16$ & 3.5 & 0.055 & 0.044 & 0.325 & 0.495 \\
\hline OBr-S1r & $0.96 \pm 0.11$ & $0.64 \pm 0.21$ & $0.67 \pm 0.22$ & 8.7 & $0.002 *$ & 0.004* & 0.008* & 0.955 \\
\hline OBr-S11 & $0.95 \pm 0.13$ & $0.67 \pm 0.15$ & $0.72 \pm 0.21$ & 6.9 & $0.006^{*}$ & $0.008^{*}$ & $0.028 *$ & 0.806 \\
\hline $\mathrm{OBr}-\mathrm{V} 2 \mathrm{r}$ & $1.0 \pm 0.0$ & $0.57 \pm 0.19$ & $0.57 \pm 0.27$ & 22.1 & $<0.001 *$ & $<0.001 *$ & $<0.001 *$ & 0.997 \\
\hline $\mathrm{OBr}-\mathrm{V} 21$ & $1.0 \pm 0.0$ & $0.60 \pm 0.12$ & $0.56 \pm 0.18$ & 39.6 & $<0.001 *$ & $<0.001 *$ & $<0.001 *$ & 0.676 \\
\hline M1r-M11 & $0.98 \pm 0.03$ & $0.92 \pm 0.11$ & $0.74 \pm 0.13$ & 12.7 & $<0.001 *$ & 0.510 & $<0.001 *$ & $0.005^{*}$ \\
\hline M1r-S1r & $1.0 \pm 0.0$ & $0.72 \pm 0.08$ & $0.68 \pm 0.08$ & 56.1 & $<0.001 *$ & $<0.001^{*}$ & $<0.001 *$ & 0.361 \\
\hline M1r-S11 & $0.99 \pm 0.01$ & $0.79 \pm 0.12$ & $0.63 \pm 0.11$ & 38.8 & $<0.001 *$ & $<0.001 *$ & $<0.001 *$ & $0.004 *$ \\
\hline M1r-V2r & $0.98 \pm 0.05$ & $0.57 \pm 0.14$ & $0.52 \pm 0.22$ & 26.5 & $<0.001 *$ & $<0.001^{*}$ & $<0.001 *$ & 0.805 \\
\hline M1r-V21 & $1.0 \pm 0.0$ & $0.54 \pm 0.12$ & $0.43 \pm 0.10$ & 116.5 & $<0.001 *$ & $<0.001^{*}$ & $<0.001 *$ & $0.010^{*}$ \\
\hline M11-S11 & $1.0 \pm 0.0$ & $0.79 \pm 0.08$ & $0.78 \pm 0.07$ & 36.4 & $<0.001 *$ & $<0.001^{*}$ & $<0.001 *$ & 0.967 \\
\hline M11-V21 & $0.99 \pm 0.03$ & $0.59 \pm 0.16$ & $0.50 \pm 0.13$ & 51.1 & $<0.001 *$ & $<0.001^{*}$ & $<0.001 *$ & 0.203 \\
\hline S1r-M11 & $1.0 \pm 0.0$ & $0.77 \pm 0.09$ & $0.56 \pm 0.10$ & 89.1 & $<0.001 *$ & $<0.001^{*}$ & $<0.001 *$ & $<0.001 \%$ \\
\hline S1r-S11 & $1.0 \pm 0.0$ & $0.68 \pm 0.09$ & $0.56 \pm 0.04$ & 148.5 & $<0.001^{*}$ & $<0.001^{*}$ & $<0.001 *$ & $<0.001 *$ \\
\hline S1r-V21 & $0.98 \pm 0.03$ & $0.68 \pm 0.17$ & $0.52 \pm 0.16$ & 36.6 & $<0.001 *$ & $<0.001^{*}$ & $<0.001 *$ & $0.032 *$ \\
\hline V2r-M11 & $1.0 \pm 0.0$ & $0.57 \pm 0.15$ & $0.46 \pm 0.13$ & 88.4 & $<0.001 *$ & $<0.001 *$ & $<0.001 *$ & $0.045^{*}$ \\
\hline V2r-S11 & $1.0 \pm 0.0$ & $0.60 \pm 0.16$ & $0.45 \pm 0.13$ & 82.3 & $<0.001 *$ & $<0.001^{*}$ & $<0.001 *$ & $0.007 *$ \\
\hline V2r-V21 & $1.0 \pm 0.0$ & $0.74 \pm 0.16$ & $0.56 \pm 0.18$ & 38.4 & $<0.001 \%$ & $<0.001 *$ & $<0.001 *$ & $0.008 *$ \\
\hline S11-V21 & $1.0 \pm 0.0$ & $0.67 \pm 0.12$ & $0.67 \pm 0.12$ & 44.9 & $<0.001 \%$ & $<0.001 *$ & $<0.001 *$ & 0.980 \\
\hline S1r-V2r & $0.96 \pm 0.10$ & $0.76 \pm 0.11$ & $0.74 \pm 0.13$ & 7.9 & $0.004 *$ & $0.013^{*}$ & 0.006* & 0.927 \\
\hline
\end{tabular}

The analysis was performed in 9 animals. The degrees of freedom were 2 (between groups) and 16 (within groups). OB, olfactory bulb; M1, primary motor cortex; S1, primary somatosensory cortex; V2, secondary visual cortex r, right; 1, left. W, wakefulness; NREM, non-REM sleep; REM, REM sleep. *, p $<0.05$. 


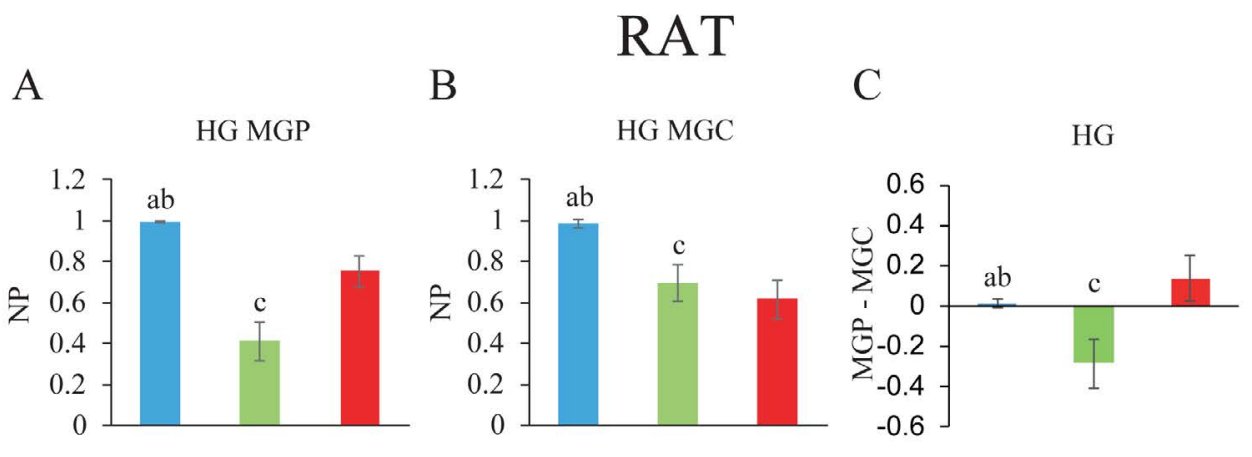

LG MGP



LG MGC

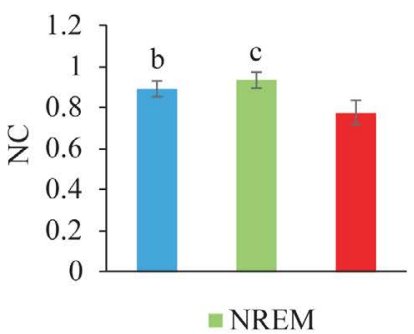

LG



Figure 4. Gamma band mean global power (MGP), mean global coherence (MGC) and their normalized difference during wakefulness and sleep in the rat. A. Low gamma (lower) and high gamma (upper) MGP. B. MGC is depicted. C. Power-coherence difference (NP-NC). The values represent the mean \pm standard deviation. Repeated measures ANOVA and Tamhane tests. a, W vs NREM sleep, $\mathrm{p}<0.05$; b, W vs REM sleep, $\mathrm{p}<0.05$; c, NREM sleep vs REM sleep, $\mathrm{p}<0.05$.

value was close to zero, it was negative during NREM sleep and positive during REM sleep (Figure 4C).

\section{Gamma activity in cats}

Dynamics of gamma activity in the cat: The dynamic changes in gamma activity in a representative cat are exhibited in Figure 5. The spectrograms and hypnograms of Figure 5A, reflect the different behavioral states of the animal. The NP for the low and high gamma bands was modified along the W-sleep cycle (Figure 5B). Pre-frontal (Pf) and parietal posterior (Pp) NP were highest during AW and decreased progressively during the transition to QW and NREM sleep. During REM sleep, there was a clear increase in the low and high gamma NP in both cortices.

We also analyzed the dynamic changes in gamma NC between Pf and Pp electrodes across behavioral states (Figure 5C). Low and high gamma NC were highest during AW and decreased during QW and NREM sleep to an intermediate value. In contrast to the NP, low and high gamma coherence during REM sleep decreased to its lowest level.

When the NP-NC function was applied (Figure 5D), during AW and QW NP-NC values were close to zero, they decreased to negatives values during NREM sleep, and inverted to positive values during REM sleep.

Gamma activity during sleep and wakefulness in the cat: The analysis in the cat was limited to two cortical areas (anterior and posterior) of the same hemisphere. In cats, the mean NP of the gamma band was different across behavioral states $\left(\mathrm{LG}, \mathrm{F}_{2,10}=132.1, \mathrm{p}<0.001\right.$; $\left.\mathrm{HG} \mathrm{Hz}, \mathrm{F}_{2,10}=36.9, \mathrm{p}<0.001\right)$. Figure $6 \mathrm{~A}$ shows that low and high gamma band power was significantly higher during $\mathrm{W}$ than during other states. The minimum level of power was recorded during NREM sleep and reached intermediate values during REM sleep.
These animals showed significant differences in the $\mathrm{NC}\left(\mathrm{LG}, \mathrm{F}_{2,10}=\right.$ 46.8, $\mathrm{p}<0.001 ; \mathrm{HG}, \mathrm{F}_{210}=56.1, \mathrm{p}<0.001$ ) (Figure 6B). During $\mathrm{W}$, low and high gamma coherence was greater and significantly different compared to the rest of the behavioral states. The lowest values for LG and HG coherence were present during REM sleep.

NP-NC was calculated in the cat. NP-NC was significantly different for the low and high gamma bands across behavioral states ( $\mathrm{LG} \mathrm{Hz}$, $\mathrm{F}_{2,10}=19.7, \mathrm{p}<0.001 ; \mathrm{HG}, \mathrm{F}_{2,10}=38.3, \mathrm{p}<0.001$ ) (Figure 6C). During W, NP-NC in LG and HG was nearly zero, while in NREM sleep it presented negative values. Positive values were observed during REM sleep.

Gamma activity during REM sleep transitions: For an in-depth examination of the transitions ( $t$ ) into and from REM sleep, we analyzed the mean gamma power and mean $z$ '-coherence in 10-second windows in cats (Figure 7) and rats (Figure 8). In both animal models, REM sleep onset was accompanied by a reduction in low and high gamma z'-coherence (Figures 7A and 8A, upper charts). Interestingly, this reduction in gamma coherence tended to precede REM sleep onset by several seconds; we considered EEG activation (desynchronization) as the beginning of REM sleep (time 0 ). In contrast, at the end of REM sleep episode, $z^{\prime}$-coherence increased (Figures 7B and 8B, upper charts).

Low and high gamma power increased during REM sleep onset (Figures 7A and 8A, lower charts). At the end of the REM sleep episodes, on average there was an increase in gamma power (reflecting W) that was followed by a decrease in this parameter (probably driven by NREM sleep) (Figures $7 \mathrm{~B}$ and $8 \mathrm{~B}$, lower charts). A short bout of W (microarousal) followed by NREM sleep, or a sustained period of W, usually followed REM sleep episodes. This phenomenon could determine a more variable low and high gamma power following REM sleep episodes. 
A
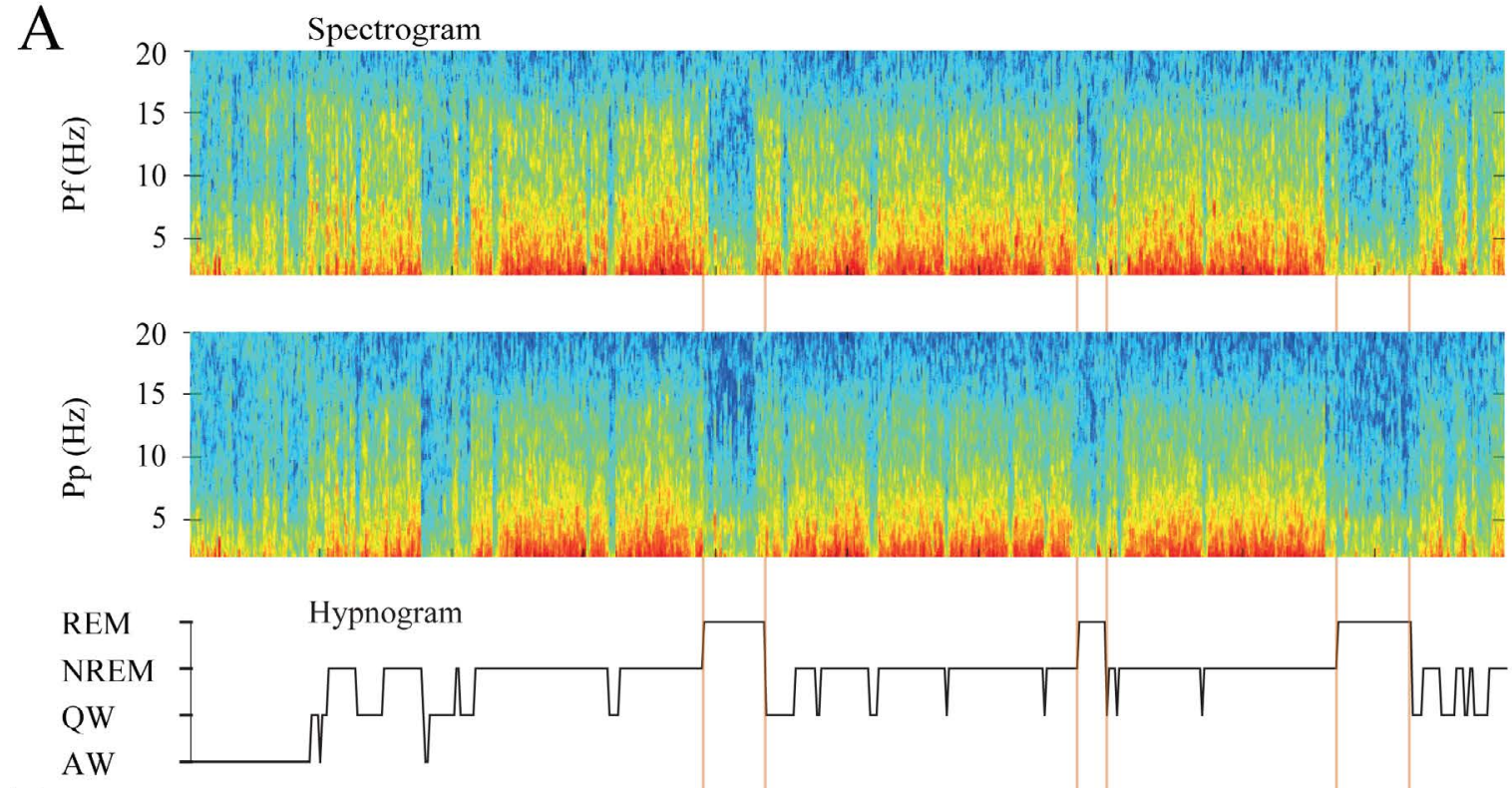

B

B
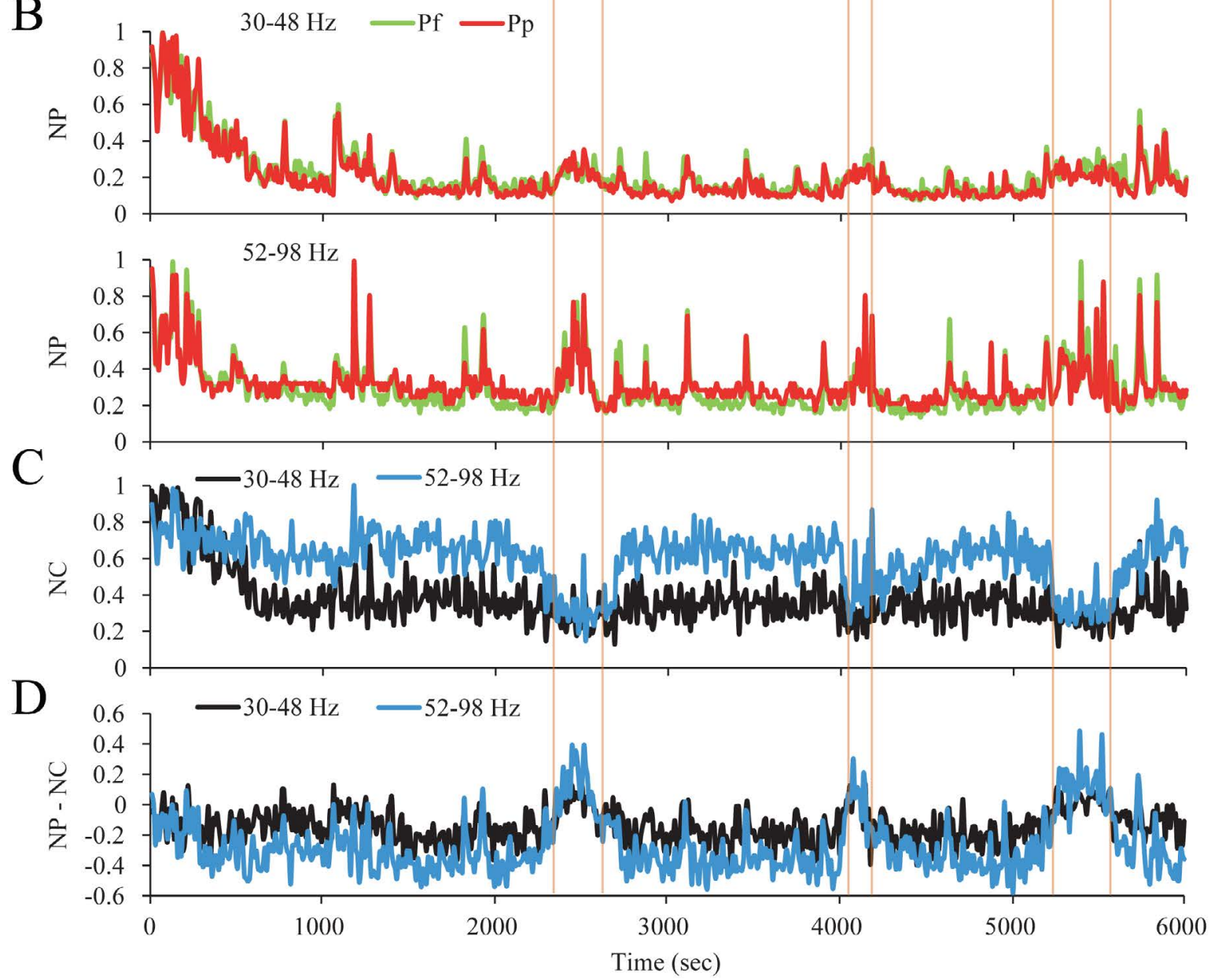

Figure 5. Dynamics of gamma activity in the cat. A. The spectrograms $(1-20 \mathrm{~Hz})$ of prefrontal (Pf) and posterior parietal (Pp) cortical recordings and the hypnogram are shown. During W and REM sleep there was weak slow wave activity. During NREM sleep, delta activity $(0.5-4 \mathrm{~Hz})$ was more prominent and there were intermittent episodes of sigma activity (9-15 Hz), which correspond to the presence of sleep spindles. Color calibration of the spectrogram is not shown. B. Normalized power (NP) of Pf (green) and Pp (red) cortices for low (upper traces) and high (lower traces) gamma bands are shown. C. Normalized z'-coherence (NC) for both gamma bands (30-48 and 52-98 Hz). D. Power-coherence difference representation (NP-NC) shows near zero values for $\mathrm{AW}$ and $\mathrm{QW}$, negative values during NREM sleep and inversion to positives values during REM sleep. All the parameters were analyzed in 10 seconds epochs. AW, alert wakefulness; QW, quiet wakefulness; NREM, NREM sleep; REM, REM sleep. 
A $\quad$ B $\quad$ CAT

HG

HG
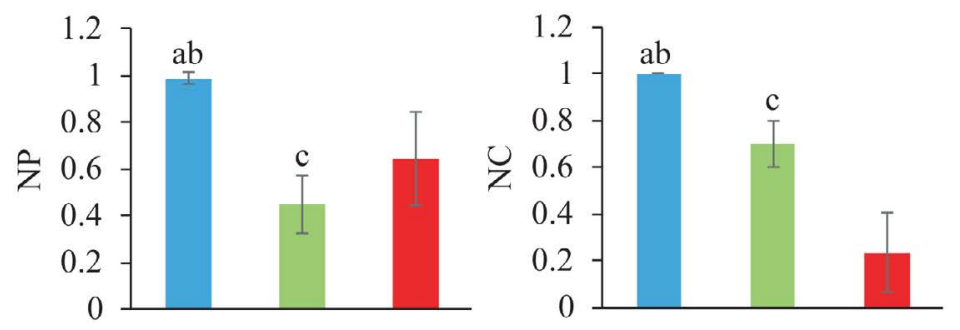

C

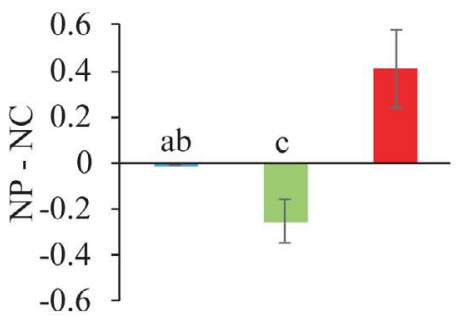

LG

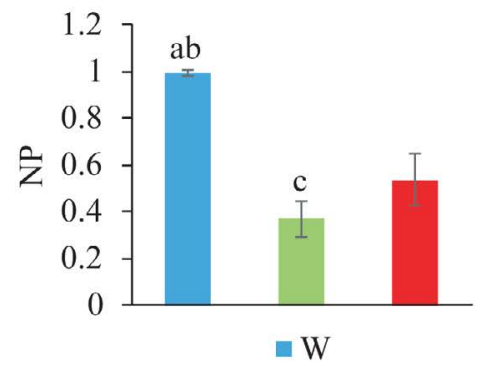

LG



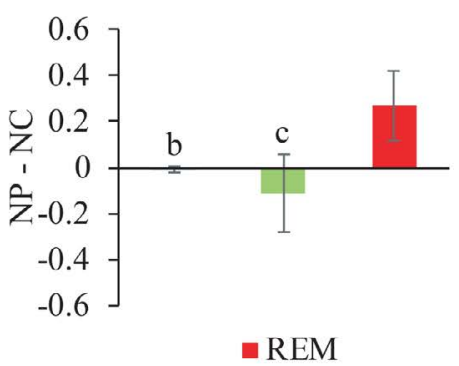

Figure 6. Gamma band normalized power (NP), normalized coherence (NC) and their normalized difference during wakefulness and sleep in the cat. A. Low gamma (lower) and high gamma (upper) mean NP of frontal (f) and posterior (p) electrodes. B. NC between both channels $\mathrm{f}$ and $\mathrm{p}$ are depicted. C. Power-coherence difference (NP-NC). The values represent the mean \pm standard deviation. Repeated measures ANOVA and Tamhane tests. a, W vs NREM sleep, $p<0.05 ; \mathrm{b}, \mathrm{W}$ vs REM sleep, $\mathrm{p}<0.05 ; \mathrm{c}, \mathrm{NREM}$ sleep, vs REM sleep, $\mathrm{p}<0.05$.

LG

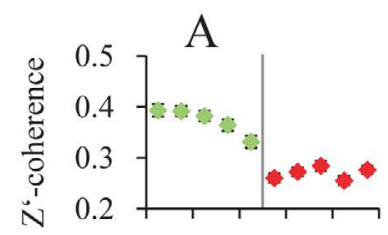

S1

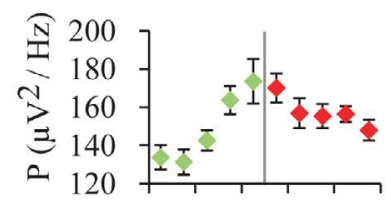

$\mathrm{V} 2$
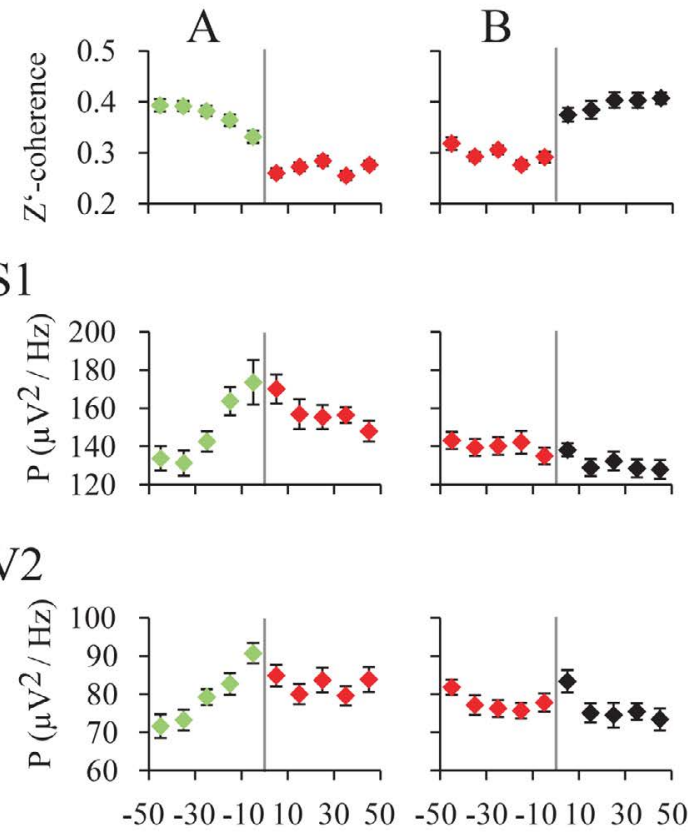

HG
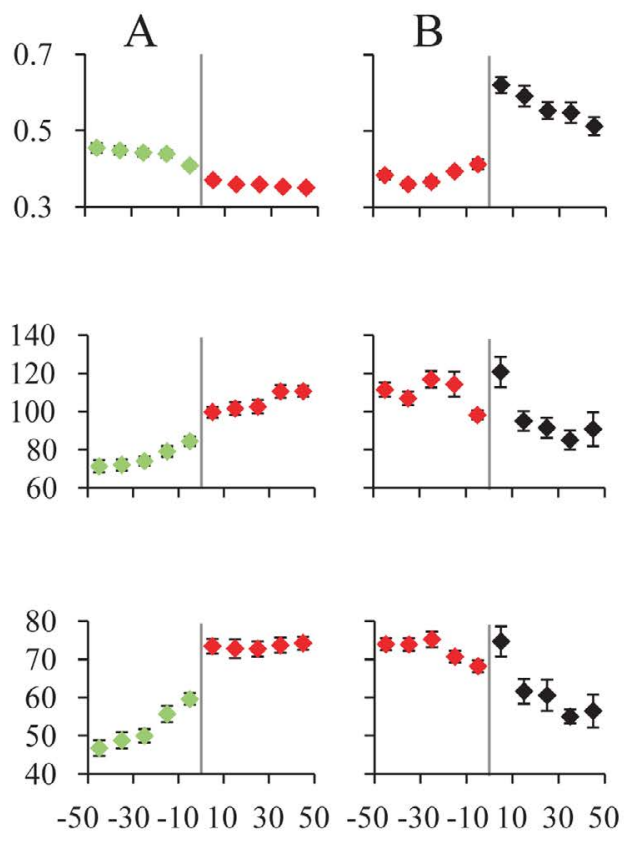

Time (sec)

Figure 7. z'-coherence and power during REM sleep transitions in the rat. A. Transition into REM sleep. The graphics depict the mean z'-coherence and power \pm standard error for $30-48$ and $52-98 \mathrm{~Hz}$, of 30 transitions of one representative rat. Data were taken from recordings of primary somatosensory (S1) and secondary visual (V2) cortices. Change of colors and the vertical line indicate the phase transition. NREM sleep episodes are symbolized in green; REM sleep episodes in red. B. Transition out of REM sleep. The mean z'-coherence and power \pm standard error for 30-48 and 52-98 Hz, of 30 transitions of the same rat are shown. Red to black transition indicates the end of the REM sleep episode. The states indicated in black were mainly micro-awakenings but some NREM sleep episodes also followed REM sleep. 
LG
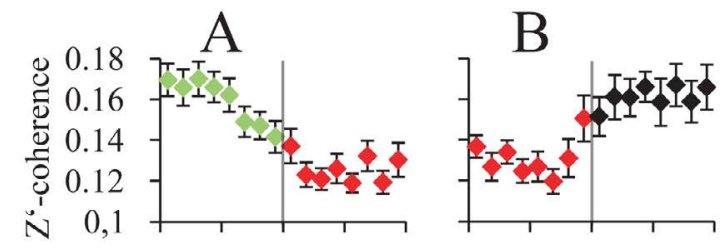

Pf
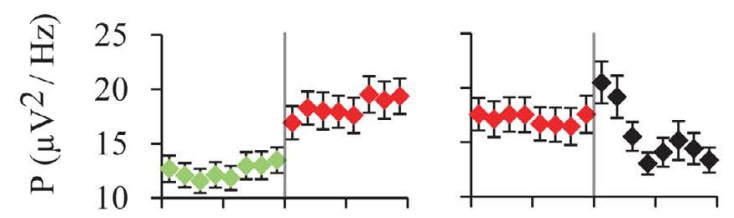

$\mathrm{Pp}$
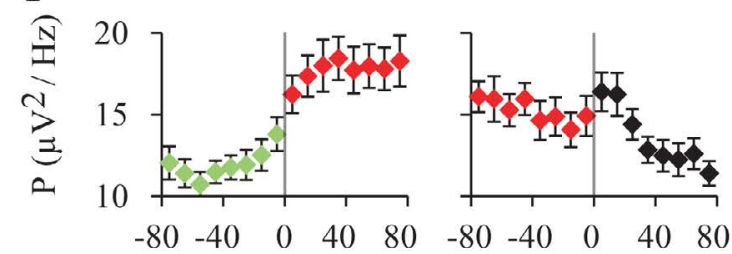

$\mathrm{HG}$
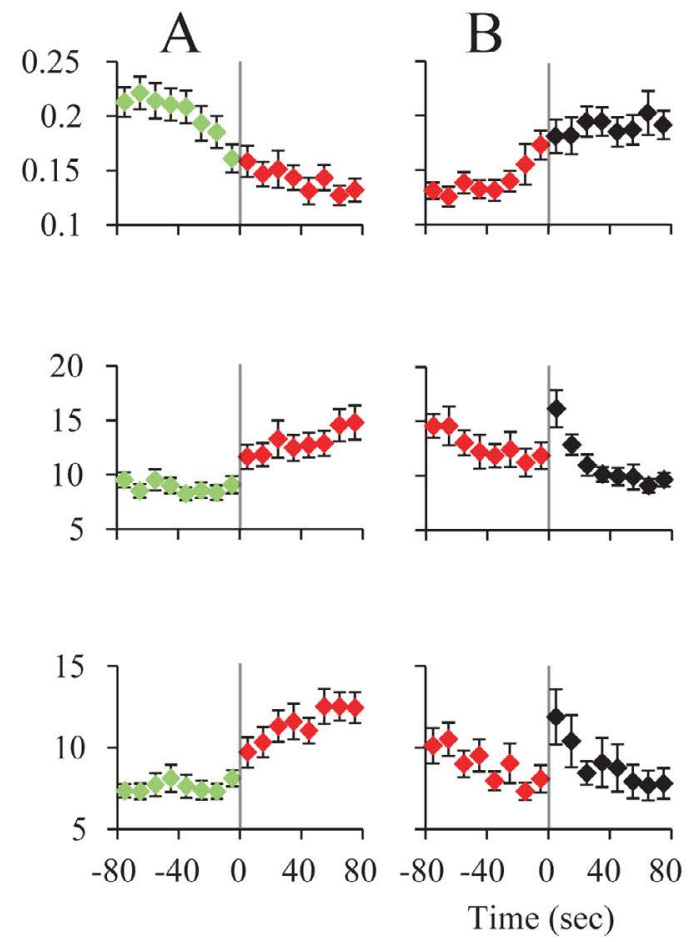

Figure 8. z'-coherence and power during REM sleep transitions in the cat. A. Transition into REM sleep. The graphics represent the mean z'-coherence and power \pm standard erro for 30-48 and 52-98 Hz, of 30 transitions of one representative cat. Data were taken from recordings of prefrontal (Pf) and posterior parietal (Pp) cortices. Change of colors and the vertical line indicate the phase transition. NREM episodes are symbolized in green; REM sleep episodes in red. B. Transition out of REM sleep. The mean z'-coherence and power \pm standard error for 30-48 and 52-98 Hz, of 30 transitions of the same cat are shown. Red to black transition indicates the end of the REM sleep episode. The states indicated in black were mainly micro-awakenings but some NREM sleep episodes also followed REM sleep.

The main relationships during $\mathrm{W}$ and sleep between gamma power, reflecting local or short-range synchronization, and gamma coherence, representing distant or large-range coupling, is schematized in Figure 9. High short (local) and long-range (distant) gamma coupling is present during $\mathrm{W}$. In contrast, high local gamma synchronization with long-range gamma uncoupling is present during REM sleep.

\section{Discussion}

In the present study, we performed a thorough analysis of gamma power and coherence in rat neocortical areas and OB; and we also performed a more spatially-limited analysis in the cat. The main result of this report is the demonstration in two different animal models (rats and cats), that during REM sleep there was a strong local (shortrange) synchronization of the neural population in both gamma (30-48 and $52-98 \mathrm{~Hz}$ ) frequency bands, while this synchronization was strongly reduced between distant areas. These data suggest that during REM sleep, in spite of a local activated state, high-frequency functional interactions between different cortical regions are lost (or highly diminished).

The relationship between local and distant gamma synchronization was further explored utilizing the gamma NP-NC function as an index (Figures 4 and 6). This index reflects the difference between the normalized power (reflecting local or short-range synchronization) and normalized coherence (distant areas or long-range synchronization); which was close to zero during $\mathrm{W}$, consisted of negative values during NREM sleep, while positive values were found during REM sleep. Therefore, this index of gamma activity was capable of differentiating between W, NREM sleep, and REM sleep both in cats and rats.
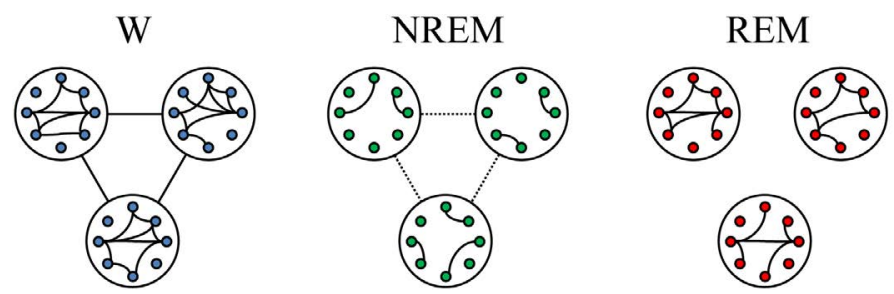

Figure 9. Schematic representation of the short and long-range gamma synchronization during wakefulness (W), NREM sleep and REM sleep. The small circles represent neurons while large circles represent the areas of the cortex where these neurons are located. Colors of neurons represent the behavioral states (blue, W; green, NREM sleep; red, REM sleep) and connecting lines between the circles represent gamma synchronization between distant cortical areas. Short-range (local) and long-range (distant) gamma synchronization occurs during W. During NREM sleep both short and long-range gamma synchronization decrease. During REM sleep, while gamma synchronization is present at local level, distant gamma coupling is absent.

\section{Gamma power and coherence during wakefulness and NREM sleep}

It is well established that gamma power and gamma intrahemispheric coherence is high during $\mathrm{W}$ in cats, rats, and humans [21$23,25,39,44]$. In the cat, gamma band coherence increases during alert $\mathrm{W}$; a fact that can be clearly observed in raw recordings [39]. Gamma coherence is also high during cataplexy (W with REM sleep atonia) induced by carbachol microinjections into the nucleus pontis oralis (NPO) of the cat [41].

In the present report, we extend previous data in rats [23], showing the cortical extent of gamma power and coherence. As shown in Figure 3 , low and high gamma power is higher in $\mathrm{W}$ than in NREM sleep and 
REM sleep in most of the neocortical areas and OB. The exemption was that low gamma power during $\mathrm{W}$ and REM sleep was similar in frontal and parietal cortices (Figure 3).

Coherence for high gamma during $\mathrm{W}$ was higher than during NREM sleep and REM sleep across all the combinations of cortical loci. However, low gamma coherence differences between W and NREM sleep varied between different cortices (Figure 4) [23].

\section{Gamma power and coherence during REM sleep}

In the present report, we demonstrated in rats and cats that gamma coherence reached a nadir during REM sleep. In the rat, high and low gamma coherence decreased in most combinations of electrodes in comparison to $\mathrm{W}$ and NREM sleep (Figure 3). However, the power in this frequency band increased compared to NREM sleep. This powercoherence relationship was also readily observed in the dynamic analysis (Figures 2 and 5), as well as in the transition into and out REM sleep (Figure 7 and 8).

This result is in accordance with the results of our previous analyses in cat [39] and rat [23]. Interestingly, gamma coherence is also lost during REM sleep induced by carbachol microinjections into the NPO of the cat [41].

In preliminary studies in human newborns, cortical gamma coherence was almost absent during REM sleep [45]. The demonstration that there is a reduction in gamma coherence between different cortical regions during REM sleep was also shown in adult humans $[20,33,46]$. In addition, Voss et al. (2009) demonstrated that gamma coherence during lucid dreaming was intermediate between $\mathrm{W}$ and REM sleep [33].

As with other EEG rhythms, gamma oscillations remain remarkably conserved in mammals irrespective of brain size [47]. The decrease in gamma coherence during REM sleep in rats, cats and humans, indicates that during this behavioral state there is a decrease in the capacity for integration across different cortices within this high frequency band, despite high local activity at these frequencies.

A recent study demonstrated that scalp fronto-temporal electrical stimulation in the lower gamma band $(\approx 40 \mathrm{~Hz})$ during REM sleep influences ongoing brain activity and induces self-reflective awareness (a feature of $\mathrm{W}$ ) in dreams (i.e., lucid dreams), while other stimulation frequencies were not effective [32]. Thus, the data support the concept that synchronous long-range oscillations of $\approx 40$ $\mathrm{Hz}$ are an electrophysiological pattern of activity that is indicative of attentive wakefulness. On the contrary, the reduction in gamma coherence during REM sleep together with increased local gamma activity (accompanied by a decrease in low frequency activity), may underlie the unique pattern of REM sleep mentation, i.e., dreams $[17,18,48]$. During NREM sleep the decrease in local and a small reduction in distant gamma coupling accompanied by an increase in low frequency oscillations (delta waves) and sleep spindles, may be the neurophysiological foundation for the reduction or absence of oneiric activity during deep NREM sleep. Interestingly, Siclari et al., (2017) have demonstrated that reports of dream experience in either REM sleep and NREM sleep were associated with decreases in delta power and increases in gamma power $(25-50 \mathrm{~Hz})$ mainly in posterior cortical regions [48].

\section{Gamma power and coherence in the $O B$ during $W$ and sleep}

It is well established that local slow field potentials in the OB are associated with breathing, and these oscillations would aid in the exchange of information between olfactory areas and other parts of the brain [49-51]. These respiratory potentials in the OB reflect respiratory rhythms during W and REM sleep, but not during NREM sleep [52], and entrain gamma oscillations in the $\mathrm{OB}$ as well as in other areas of the brain $[11,14,15,53]$.

Our data show that in the $\mathrm{OB}$, the maximum gamma power is present during $\mathrm{W}$, decreases during NREM sleep, and increases to intermediate values during REM sleep. LG coherence between OB and most neocortical areas did not differ across behavioral states (Figure 3 and Table 2). However, HG coherence between the OB and medial and posterior areas of neocortex was higher during $\mathrm{W}$ than sleep (Figure 3 and Table 3). New studies are needed to shed light in the functional interrelation between the $\mathrm{OB}$ and neocortical areas during $\mathrm{W}$ and sleep.

\section{Gamma coherence and the waking-promoting systems}

Cognitive activity and different EEG rhythms are generated by the activity of cortical and thalamic neurons, which are reciprocally connected [29,54]. Gamma-band rhythmogenesis is also inextricably tied to perisomatic inhibition in the neocortex, wherein the key ingredient is $\mathrm{GABA}_{\mathrm{A}}$ receptor-mediated inhibition [12]. However, both neocortical gamma power and coherence during $\mathrm{W}$ and sleep are modulated by the activating or waking-promoting systems of the brainstem, hypothalamus and basal forebrain that directly or indirectly project to the thalamus and/or cortex $[55,56]$. By regulating thalamocortical activities, these activating systems produce electrographic and behavioral arousal.

The activating systems decrease their activity during the NREM sleep. However, the activity of the various components of these systems differs markedly during REM sleep. While most monoaminergic systems decrease their firing rate during REM sleep (REM-off neurons), cholinergic neurons increase their discharge during this behavioral state (REM-on neurons), which contributes to the cortical activation $[55,56]$. Therefore, it is expected that cholinergic REM-on neurons, whose soma are located in the mesopontine and basal forebrain region, may contribute in the promotion of local gamma synchronization (gamma power). In addition, because these cholinergic neurons turn on during REM sleep, they should not be critical to the generation of gamma coherence (distant coupling), which is absent during this state. In fact, systemic muscarinic antagonists do not block gamma coherent activity [57].

Noradrenergic, serotonergic and histaminergic neurons that are active during $\mathrm{W}[55,56]$, may be crucial in promoting gamma coherence during this behavioral state. Their lack of activity during REM sleep may be involved in the absence of gamma coherence during this state.

Other neuronal systems, such as hypocretinergic and dopaminergic neurons that are active during $\mathrm{W}$, as well as GABAergic or glutamatergic neurons which are located in the mesopontine reticular formation and basal forebrain, may also contribute to the profile of gamma activity during $\mathrm{W}$ and sleep [55,56]. In fact, Kim et al., [58], highlighted the role of cortical-projecting GABAergic neurons of the basal forebrain in the generation of gamma oscillations in the EEG [58]. In addition, the authors suggest that cholinergic neurons within this area are not critical for the generation of these oscillations.

It is important to note that firing within the gamma band range is present in different sites of the reticular activating system, including the pedunculo-pontine tegmental nucleus (PPN) [59]. In this area, gamma oscillations are modulated by two independent pathways related to different $\mathrm{Ca} 2+$ channel types which suggests different ways 
of modulating waking and REM sleep [60,61]. This subcortical gamma activity possibly contribute to "bottom up" neocortical gamma activity during $\mathrm{W}$ and REM sleep [62].

\section{Local and distant gamma coupling relationship}

The NP-NC index clearly shows that short and long-range gamma synchronization is deeply modified across behavioral states; this fact is illustrated in the model presented in Figure 9. As discussed above, this electrophysiological phenomenon could be related to the cognitive features that characterize W, NREM sleep and REM sleep. Moreover, NREM sleep and REM sleep dream content, and different drugs or processes that affect cognition, must all modify the NP-NC index. We hypothesize that dissociative drugs such as ketamine, or psychiatric conditions such as psychosis, will also increase the index values as in REM sleep. Finally, this NP-NC index, that signals the presence of REM sleep, should be applied to confirm the absence of REM sleep in aquatic mammals, that for its obligate swimming behavior during sleep do not have REM sleep atonia [63-65].

\section{Conclusions}

During REM sleep, despite a locally activated EEG, there is uncoupling of gamma frequency oscillations between distant neocortical sites. In spite of regional variations, this gamma activity pattern extends, in rats, from the posterior cortices to the OB. Therefore, although local gamma coupling is similar to $\mathrm{W}$, functional interactions among different cortical area, which are critical for cognitive functions, are radically different during REM sleep. Since this feature is conserved in rats, cats and likely in humans, we consider that this short-range gamma coupling along with gamma long-range uncoupling during REM sleep is a defining trait of REM sleep in mammals.

\section{Acknowledgement}

This study was partially supported by a grant of the Comisión Sectorial de Investigación Científica (CSIC) and the "Programa de Desarrollo de Ciencias Básicas, PEDECIBA". S. Castro, A. Mondino and M. Cavelli currently have a postgraduate fellowship from the Agencia Nacional de Investigación en Innovación (ANII) and Comisión de Apoyo a Postgrados (CAP), respectively.

\section{Competing interests}

All the authors declare no conflict of interest.

\section{Author contributions}

Financial support. P.T., A.F., M.C.

Experimental design. M.C., P.T., S.CZ.

Experimental procedures. M.C., S.CZ., A.M., J.G., P.T.

Analysis of the data. M.C., S.CZ., A.M., J.G.

Discussion and interpretation of the data. M.C., P.T., S.CZ., A.M., J.G., A.F.

Wrote the manuscript. M.C., P.T.

All the authors participated in critical revision the manuscript, added important intellectual content, and approved the definitive version.

\section{References}

1. Singer W (2015) Complexity as Substrate for Neuronal Computations. Acad Sci. Pontif. City, Vatican. PP: 1-10.
2. Singer W (2007) Binding by synchrony. Scholarpedia 2: 1657.

3. von der Malsburg C, Schneider W (1986) A neural cocktail-party processor. Biol Cybern 54: 29-40. [Crossref]

4. von der Malsburg C (1995) Binding in models of perception and brain function. Curr Opin Neurobiol 5: 520-526. [Crossref]

5. Singer W (1999) Neuronal Synchrony?: A Versatile Code for the Definition of Relations?? Most of our knowledge about the functional organization. Neuron 24: 4965 .

6. Varela F, Lachaux JP, Rodriguez E, Martinerie J (2001) The brainweb: phase synchronization and large-scale integration. Nat Rev Neurosci 2: 229-239. [Crossref]

7. Eckhorn R, Bauer R, Jordan W, Brosch M, Kruse W, et al. (1988) Coherent oscillations: a mechanism of feature linking in the visual cortex? Multiple electrode and correlation analyses in the cat. Biol Cybern 60: 121-130. [Crossref]

8. Gray CM, König P, Engel AK, Singer W (1989) Oscillatory responses in cat visua cortex exhibit inter-columnar synchronization which reflects global stimulus properties. Nature 338: 334-337.

9. Fries P (2005) A mechanism for cognitive dynamics: neuronal communication through neuronal coherence. Trends Cogn Sci 9: 474-480. [Crossref]

10. Jasper HH, Andrews HL (1938) Brain potentials and voluntary muscle activity in man J Neurophysiol 1: 87-100.

11. Adrian ED (1942) Olfactory reactions in the brain of the hedgehog. $J$ Physiol 100 459-473. [Crossref]

12. Buzsáki G, Wang XJ (2012) Mechanisms of gamma oscillations. Annu Rev Neurosci 35: 203-225. [Crossref]

13. Rieder MK, Rahm B, Williams JD, Kaiser J (2011) Human $\hat{\mathrm{I}}^{3}$-band activity and behavior. Int J Psychophysiol 79: 39-48. [Crossref]

14. Rojas-Líbano D, Kay LM (2008) Olfactory system gamma oscillations: the physiological dissection of a cognitive neural system. Cogn Neurodyn 2: 179-194.

15. Freeman WJ, Schneider W (1982) Changes in spatial patterns of rabbit olfactory EEG with conditioning to odors. Psychophysiology 19: 44-56. [Crossref]

16. Uhlhaas PJ, Pipa G, Lima B, Melloni L, Neuenschwander S, et al. (2009) Neural synchrony in cortical networks?: history, concept and current status. Front Integr Neurosci 3: 1-19.

17. Hobson JA (2009) REM sleep and dreaming: towards a theory of protoconsciousness. Nat Rev Neurosci 10: 803-813. [Crossref]

18. Nir Y, Tononi G (2010) Dreaming and the brain: from phenomenology to neurophysiology. Trends Cogn Sci 14: 88-100. [Crossref]

19. Gottesmann C (2006) The dreaming sleep stage: a new neurobiological model of schizophrenia? Neuroscience 140: 1105-1115. [Crossref]

20. Cantero JL, Atienza M, Madsen JR, Stickgold R (2004) Gamma EEG dynamics in neocortex and hippocampus during human wakefulness and sleep. Neuroimage 22: 1271-1280. [Crossref]

21. Llinás R, Ribary U (1993) Coherent $40-\mathrm{Hz}$ oscillation characterizes dream state in humans. Proc Natl Acad Sci U S A 90: 2078-2081. [Crossref]

22. Maloney KJ, Cape EG, Gotman J, Jones BE (1997) High-frequency gamma electroencephalogram activity in association with sleep-wake states and spontaneous behaviors in the rat. Neuroscience 76: 541-555.

23. Cavelli M, Castro S, Schwarzkopf N, Chase MH, Falconi A, et al. (2015) Coherent neocortical gamma oscillations decrease during REM sleep in the rat. Behav Brain Res 281: $318-325$

24. Buzsáki G, Anastassiou CA, Koch C (2012) The origin of extracellular fields and currents--EEG, ECoG, LFP and spikes. Nat Rev Neurosci 13: 407-420. [Crossref]

25. Chrobak JJ, Buzsáki G (1998) Gamma oscillations in the entorhinal cortex of the freely behaving rat. J Neurosci 18: 388-398. [Crossref]

26. Bullock TH, McClune MC (1989) Lateral coherence of the electrocorticogram: a new measure of brain synchrony. Electroencephalogr Clin Neurophysiol 73: 479-498. [Crossref]

27. Bullock TH, McClune MC, Achimowicz JZ, Iragui-Madoz VJ, Duckrow RB, et al (1995) Temporal fluctuations in coherence of brain waves. Proc Natl Acad Sci U S A 92: 11568-11572. [Crossref] 
28. Cantero JL, Atienza M, Salas RM (2000) Valor clínico de la coherencia EEG como índice electrofisiológico de conectividad córtico-cortical durante el sueño. Rev Neurol 31: $442-454$.

29. Llinás R, Ribary U, Contreras D, Pedroarena C (1998) The neuronal basis for consciousness. Philos Trans R Soc Lond B Biol Sci 353: 1841-1849. [Crossref]

30. Hadjipapas A, Hillebrand A, Holliday IE, Singh KD, Barnes GR (2005) Assessing interactions of linear and nonlinear neuronal sources using MEG beamformers: a proof of concept. Clin Neurophysiol 116: 1300-1313. [Crossref]

31. Melloni L, Molina C, Pena M, Torres, W. Singer, E. Rodriguez (2007) Synchronization of neural activity across cortical areas correlates with conscious perception. $J$ Neurosci 27: 2858-2865.

32. Voss U, Holzmann R, Hobson A, Paulus W, Koppehele-Gossel J, et al. (2014) Induction of self awareness in dreams through frontal low current stimulation of gamma activity. Nat Neurosci PP: 1-5.

33. Voss U, Holzmann R, Tuin I, Hobson JA (2009) Lucid dreaming: a state of consciousness with features of both waking and non-lucid dreaming. Sleep 32: 11911200. [Crossref]

34. John ER (2002) The neurophysics of consciousness. Brain Res Brain Res Rev 39: 1-28. [Crossref]

35. Mashour GA (2006) Integrating the science of consciousness and anesthesia. Anesth Analg 103: 975-982. [Crossref]

36. Pal D, Silverstein BH, Lee H, Mashour GA (2016) Neural Correlates of Wakefulness, Sleep, and General Anesthesia: An Experimental Study in Rat. Anesthesiology 125: 929-942. [Crossref]

37. Uhlhaas PJ, Singer W (2010) Abnormal neural oscillations and synchrony in schizophrenia. Nat Rev Neurosci 11: 100-113. [Crossref]

38. Sun Y, Farzan F, Barr MS, Kirihara K, Fitzgerald PB, et al. (2011) $\hat{\mathrm{I}}^{3}$ oscillations in schizophrenia: mechanisms and clinical significance. Brain Res 1413: 98-114. [Crossref]

39. Castro-Zaballa S, Falconi A, Chase MH, Torterolo P (2013) Coherent neocortical 40$\mathrm{Hz}$ oscillations are not present during REM sleep. Eur J Neurosci 37: 1330-1339.

40. Castro-Zaballa S, Cavelli M, Vollono P, Chase MH, Falconi A, et al. (2014) Interhemispheric coherence of neocortical gamma oscillations during sleep and wakefulness. Neurosci Lett 578: 197-202.

41. Torterolo P, Castro-Zaballa S, Cavelli M, Chase MH, Falconi A (2016) Neocortical $40 \mathrm{~Hz}$ oscillations during carbachol-induced rapid eye movement sleep and cataplexy. Eur J Neurosci 43: 580-589.

42. Bullock TH, Buzsáki G, McClune MC (1990) Coherence of compound field potentials reveals discontinuities in the CA1-subiculum of the hippocampus in freely-moving rats. Neuroscience 38: 609-619.

43. Benedetto L, Rodriguez-Servetti Z, Lagos P, D’Almeida V, Monti JM, et al. (2013) Microinjection of melanin concentrating hormone into the lateral preoptic area promotes non-REM sleep in the rat. Peptides 39: 11-15.

44. Steriade M, Amzica F, Contreras D (1996) Synchronization of fast $(30-40 \mathrm{~Hz})$ spontaneous cortical rhythms during brain activation. J Neurosci 16: 392-417.

45. Castro S, Cavelli M, Torterolo P, Falconi A, Rava M, et al. (2014) Congr Int Neuropediatría Colonia.

46. Pérez-Garci E, del-Río-Portilla Y, Guevara M, Arce C, Corsi-Cabrera M (2001) Paradoxical sleep is characterized by uncoupled gamma activity between frontal and perceptual cortical regions. Sleep 24: 118-126.
47. Buzsáki G, Logothetis N, Singer W (2013) Scaling brain size, keeping timing: Evolutionary preservation of brain rhythms. Neuron 80 : 751-764.

48. Siclari F, Baird B, Perogamvros L, et al. (2017) The neural correlates of dreaming. Nat Neurosci 20: 872-878. [Crossref]

49. Rojas-Líbano D, Frederick DE, Egaña JI, Kay LM (2014) The olfactory bulb thet rhythm follows all frequencies of diaphragmatic respiration in the freely behaving rat Front Behav Neurosci 8: 214

50. Lockmann AL, Laplagne DA, Leão RN, Tort AB (2016) A Respiration-Coupled Rhythm in the Rat Hippocampus Independent of Theta and Slow Oscillations. $J$ Neurosci 36: 5338-5352. [Crossref]

51. Nguyen Chi V, Muller C, Wolfenstetter T, Yanovsky Y, Draguhn A, et al. (2016) Hippocampal Respiration-Driven Rhythm Distinct from Theta Oscillations in Awake Mice. J Neurosci 36: 162-177.

52. Jessberger J, Zhong W, Brankack J, Draguhn A (2016) Olfactory Bulb Field Potentials and Respiration in Sleep-Wake States of Mice. Neural Plast 2016: 1-9.

53. Zhong W, Ciatipis M, Wolfenstetter T, Jessberger J, Müller C, et al. (2017) Selective entrainment of gamma subbands by different slow network oscillations. Proc Natl Acad Sci U.S. A..

54. Llinás R, Ribary U (2001) Consciousness and the brain. The thalamocortical dialogue in health and disease. Ann N Y Acad Sci 929: 166-175. [Crossref]

55. Torterolo P, Vanini G (2010) [New concepts in relation to generating and maintaining arousal]. Rev Neurol 50: 747-758. [Crossref]

56. Torterolo P, Monti JM, Pandi-Perumal S (2016) Synposis Sleep Med., Apple Academic Press, pp. 1-22.

57. Castro s, Cavelli M, Falconi A, Torterolo P (2016) $23^{\text {rd }}$ Congr Eur Sleep Res Soc Bol, Italy.

58. Kim T, Thankachan S, McKenna JT, McNally JM, Yang C, et al. (2015) Cortically projecting basal forebrain parvalbumin neurons regulate cortical gamma band oscillations. Proc Natl Acad Sci 201413625

59. Garcia-Rill E, Luster B, D’Onofrio S, Mahaffey S, Bisagno V, et al. (2016) Implication of gamma band activity in the pedunculopontine nucleus. J Neural Transm (Vienna) 123: 655-665. [Crossref]

60. Luster B1, D’Onofrio S, Urbano F, Garcia-Rill E (2015) High-threshold Ca2+ channels behind gamma band activity in the pedunculopontine nucleus (PPN). Physiol Rep 3 [Crossref]

61. Luster br, Urbano FJ, Garcia-rill E (2016) Intracellular mechanisms modulating gamma band activity in the pedunculopontine nucleus (PPN). Physiol Rep 4: 1-16.

62. Garcia-Rill E (2017) Bottom-up gamma and stages of waking. Med Hypotheses 104 58-62. [Crossref]

63. Lyamin OI, Kosenko PO, Lapierre JL, Mukhametov LM, Siegel JM (2008) Fur seals display a strong drive for bilateral slow-wave sleep while on land. $J$ Neurosci 28 : 12614-12621. [Crossref]

64. Lyamin OI, Manger PR, Ridgway SH, Mukhametov LM, Siegel JM (2008) Cetacean sleep: an unusual form of mammalian sleep. Neurosci Biobehav Rev 32: 1451-1484. [Crossref]

65. Madan V, Jha SK (2012) Sleep alterations in mammals: did aquatic conditions inhibit rapid eye movement sleep? Neurosci Bull 28: 746-758. [Crossref]

Copyright: $(02017$ Cavelli M. This is an open-access article distributed under the terms of the Creative Commons Attribution License, which permits unrestricted use, distribution, and reproduction in any medium, provided the original author and source are credited. 